\title{
Acute nigro-striatal blockade alters cortico-striatal encoding: An in vivo electrophysiological study
}

\author{
Chiara Prosperetti ${ }^{\text {a,c }}$, Giuseppe Di Giovanni ${ }^{b}$, Alessandro Stefani ${ }^{\text {c }}$, Jens C. Möller ${ }^{\text {a }}$, Salvatore Galati ${ }^{\text {a,* }}$ \\ a Experimental Laboratory, Neurocenter of Southern Switzerland, Lugano, Switzerland \\ b Department of Physiology and Biochemistry, University of Malta, Malta \\ c Department of Neurology, University of Rome "Tor Vergata”, Italy
}

\section{A R T I C L E I N F O}

\section{Article history:}

Received 21 December 2012

Revised 21 February 2013

Accepted 18 March 2013

Available online $\mathrm{xxxx}$

\section{Keywords:}

Parkinson's disease

Basal ganglia synchronization

Striatum

Acute dopamine depletion

Cortical local field potentials

\begin{abstract}
A B S T R A C T
Spreading of slow cortical rhythms into the basal ganglia (BG) is a relatively well-demonstrated phenomenon in the Parkinsonian state, both in humans and animals. Accordingly, striatal dopamine (DA) depletion, either acute or chronic, drives cortical-globus pallidus (GP) and cortical-substantia nigra pars reticulata (SNr) slow wave coherences in urethane-anesthetized rats. This paper investigates the striatal dynamics following acute DA depletion by tetrodotoxin (TTX) injection in the medial forebrain bundle (MFB) with respect to the transmission of slow cortical rhythms throughout the BG in more detail. The acute DA depletion offers the advantage of detecting electrophysiological changes irrespectively of chronically developing compensatory mechanisms. We observed that the acute blockade of the dopaminergic nigro-striatal pathway reshapes the firing rate and pattern of the different striatal neuron subtypes according to cortical activity, possibly reflecting a remodeled intrastriatal network. The observed alterations differ amongst striatal neuronal subtypes with the striatal medium spiny neurons and fast-spiking neurons being the most affected, while the tonically active neurons seem to be less affected. These acute changes might contribute to the diffusion of cortical activity to BG and the pathophysiology of Parkinson's disease (PD).
\end{abstract}

(C) 2013 Elsevier Inc. All rights reserved.

\section{Introduction}

Micro-electrode recordings during functional neurosurgery in PD have allowed the demonstration of abnormally synchronized oscillatory activity at multiple structures of the BG-cortical loop (Brown, 2003; Hammond et al., 2007). This excessive synchronization might represent an electrophysiological trait of the disease being directly correlated to the clinical state and susceptible to levodopa therapy or deep-brain stimulation (DBS; Eusebio et al., 2011; Kühn et al., 2006). Similar findings have been observed in chronically as well as in acutely dopaminedepleted animals (Fuentes et al., 2009; Galati et al., 2009, 2010; Magill et al., 2001; Sharott et al., 2005). In these animals, cortical slow wave activity (SWA) spreads into the BG network providing evidence of a perturbed mechanism of cortical input processing in the dopamine-

Abbreviations: PD, Parkinson's disease; BG, basal ganglia; DA, dopamine; MFB, medial forebrain bundle; TTX, tetrodotoxin; ECoG, electrocorticogram; SWA, slow wave activity; SNr, substantia nigra pars reticulate; STN, nucleus subthalamicus; SNc, substantia nigra pars compacta; ISI, inter-spike interval; CV, coefficient of variation; AutoCrl, autocorrelograms; MSN, medium spiny neuron; FSI, fast-spiking interneuron; TAN, tonically active large aspiny interneuron.

* Corresponding author at: Experimental Laboratory, Neurocenter of Southern Switzerland, Via Tesserete 46, 6903 Lugano, Switzerland. Fax: +41 918116915.

E-mail address: salvatore.galati@eoc.ch (S. Galati). depleted status (Fuentes et al., 2009; Galati et al., 2009, 2010; Magill et al., 2001). These features have been ascribed to changes of intrinsic voltage-gated conductance in the nucleus subthalamicus (STN) (Beurrier et al., 1999; Nambu and Llinaś, 1994) and/or to an impaired interplay between the STN and the GP, leading to oscillatory behavior (Plenz and Kitai, 1999). In agreement with these data, we recently described that intra-GP haloperidol or bicuculline injection generates cortical-GP synchronization supporting the presence of local mechanisms in pathological oscillatory neuronal behavior (Galati et al., 2009).

Besides, several lines of evidence have suggested an involvement of the striatum in the pathogenesis of excessive synchronization in PD, probably due to an enhanced corticostriatal glutamatergic excitatory drive and/or to decreased interneuronal inhibition. Three different neuronal cell types in the striatum may be implicated in this electrophysiological phenomenon. It has been quite consistently proven that the activity of the GABAergic medium spiny neurons (MSN) is increased (Blume et al., 2009; Galarraga et al., 1986; Liang et al., 2008; Tang et al., 2001; Tseng et al., 2001; Zold et al., 2012). Accordingly, abnormal MSN hyperactivity and synchronization in 6-OHDA animals is reduced by intrastriatal blockade of glutamatergic transmission. However, also the application of the $\mathrm{GABA}_{\mathrm{A}}$ receptor blocker bicuculline facilitated the electrophyiological consequences of dopamine depletion indicating decreased interneuronal inhibition and hypothetically reflecting a reduced activity of the GABAergic fast-spiking interneurons (FSI) (Carrillo-Reid et al., 2008; Costa et al., 2006; Jáidar et al., 2010; Taverna et al., 2008; 
Tecuapetla et al., 2009). These GABAergic interneurons show a coordinated high-frequency firing coherent with the cortex in normal animals (Sharott et al., 2009). However, in the chronic DA depletion state the activity of the FSI is rather spared. FSI provide a strong feed-forward inhibition upon the MSN and their stable activity in the DA depletion state worsened the striatal imbalance between MSN projecting to the GP or the substantia nigra pars reticulata (Mallet et al., 2006). The GABAergic effect may also be explained by increased interneuronal inhibition of MSN that conversely leads to an increased activity of these neurons (McCarthy et al., 2011). Besides the cholinergic tonically active large aspiny interneurons (TAN) have to be taken into consideration as proposed by the intrastriatal infusion of the cholinergic agonist carbachol (McCarthy et al., 2011).

So far, the role of the diverse systems controlling the MSN activity remains poorly defined and has been investigated in a severe chronic DA depletion state in which many histological alterations have already occurred. The real impact of the substantia nigra pars compacta (SNc) DA system upon the striatum and its consequences on the BG network is not well documented. To address this question we adopted a model based on an acute block of the medial forebrain bundle (MFB) by tetrodotoxin (TTX). In previous observations we recently showed that TTX-induced MFB impairment considerably changes the interaction between STN and GP and the BG output (Galati et al., 2009, 2010).

\section{Methods}

Animals

Experimental procedures were carried out on 49 adult male Wistar rats weighing 250-300 g in compliance with Swiss laws on animal experimentation and with the National Institute of Health's Guide for the Care and Use of Laboratory Animals.

\section{Surgery}

Rats were anesthetized with urethane $(1.4 \mathrm{~g} / \mathrm{kg}$, i.p.) (Sigma Chemical Co., St. Louis, MO, USA) and mounted on a stereotaxic instrument (Stoelting Co., Wheat Lane Wood Dale, IL, USA). Body temperature was maintained at $37-38{ }^{\circ} \mathrm{C}$ with a heating pad placed beneath the animal. A midline scalp incision was made and the skull was almost completely drilled on the left side or both. The dura was then removed to expose the cortical surface. All wound margins were infiltrated with a local anesthetic (bupivacaine).

\section{Electrophysiology}

The electrophysiological methods are extensively described elsewhere (Galati et al., 2009). Briefly, electrocorticogram (ECoG) recordings coupled with striatal single unit extracellular recordings were performed. The ECoG was recorded via silver chloride screw electrodes placed on the cortical surface above the ipsilateral frontal cortex (3.0 $\mathrm{mm}$ anterior of the bregma and 2.0 lateral to the midline) and referenced against an indifferent electrode. Raw EEG was band-passfiltered (0.1-100 Hz), amplified (2000×; model 12A5 amplifier, Grass Instrument Company, Quincy, MA), sampled $(1000 \mathrm{~Hz})$ on-line and stored on a computer connected to an analog/digital interface (micro1401 mk II, Cambridge Electronic Design, Cambridge, UK). During ECoG recording, extracellular action potentials of striatal neurons were acquired using $\sim 15 \mathrm{M} \Omega$ glass electrodes (tip diameter $\sim 1.5 \mu \mathrm{m}$ ) containing saline solution $(2 \mathrm{M} \mathrm{NaCl})$. Electrode signals were amplified (10.000×; ISO-DAM8; World Precision Instruments, Hertfordshire, UK), band-pass filtered (300-1000 Hz), sampled (60 kHz) on-line and stored on a computer connected to the Cambridge Electronic Design (CED) 1401 interface (see Galati et al., 2006, 2008, 2009, 2010).

\section{Pharmacological blockade of the MFB}

During ECoG and extra-cellular sampling, TTX ( $5 \mu \mathrm{M}$ in $\mathrm{NaCl})$ was infused in the MFB (stereotaxic coordinates: $2.56 \mathrm{~mm}$ posterior to the bregma, $2 \mathrm{~mm}$ lateral to the midline, and $8.6 \mathrm{~mm}$ below the cortical surface; Figs. 1A, B) by a 30 gauge stainless steel tube (external $\varnothing$ $0.2 \mathrm{~mm}$ ) connected via a tubing to a $25 \mu \mathrm{l}$ pump-driven syringe (CMA 400 syringe pump) at an infusion rate of infusion rate of $1 \mu \mathrm{min}^{-1}$ for 2-5 min (Galati et al., 2009, 2010).

\section{Data analysis}

Single-unit activity and ECoG were analyzed off-line by Spike 2 software (CED, Cambridge, UK). During urethane-induced deep anesthesia frontal ECoG was characterized by regularly occurring slow-waves of large amplitude $(>500 \mu \mathrm{V})$ in which a smaller and faster activity $(<200 \mu \mathrm{V})$ overlaid specific portions (Galati et al., 2009, 2010; Magill et al., 2000; Steriade et al., 1993). ECoG was assessed and epochs of robust cortical SWA were identified before and after MFB injection in conjunction with a portion of the coincident striatal spike trains of 500 events. These spikes were utilized for the subsequent characterization of cellular subtypes. Spike sorting was made by applying a principal component analysis in order to select the spikes belonging to a putative neuron subtype. We used several parameters from the average waveform of the collected spikes. Specifically, we measured the total and peak amplitude and the total and peak duration. On the basis of K-means analysis we identified three clearly separate neuronal subtypes by using the peak/total amplitude ratio and peak length (Fig. 1).

The inter-spike interval (ISI) and related parameters such as the mean ISI and its reciprocal, mean firing rate, coefficient of variation (CV), skewness and kurtosis were further analyzed. Autocorrelograms (AutoCrl) were used to detect the rhythmic neuronal activity by plotting $2000 \mathrm{~ms}$ intervals with $1 \mathrm{~ms}$ bin width. Phase histograms were constructed by using the corresponding Spike 2 (CED) script in order to determine the relationship between spike discharge and ECoG. The phase histogram was used to show how striatal spikes were distributed with respect to the cyclical process identified by the peaks of SWA (60 bins). The phase histogram indicates the firing probability of a neuron with regard to the ECoG. The correlation was recognized by putting a threshold level above noise (mean threshold SD, $3.2 \pm 1.2$ ) with a visually pre-set width ranging between 0.5 and $2 \mathrm{~s}$.

\section{Statistics}

Statistical analysis was performed by using a statistical software (IBM SPSS). Statistical comparisons of firing rates and ISI parameters were conducted using the Mann-Whitney U-test. The comparison within the values of each phase ( 60 bins, from 0 to $360^{\circ}$ ) was performed using the non-parametric Friedman ANOVA whilst the comparison between the power of coherence of pre-TTX and post-TTX was performed by the Mann-Whitney U-test. The Bonferroni correction was applied for the multiple (60 bins) comparison leading the $P$ value threshold up to 0.0008 .

\section{Results}

\section{Electrophysiological classification of striatal neuronal types}

We recorded the extracellular activity of striatal neurons from forty-nine rats. The spike waveform of all cells $(n=86)$ recorded in the striatum showed a waveform characterized by a biphasic $(-/+)$ action potential. As previously described, two neuronal types were clearly distinguishable on the basis of discharge frequency pattern (Galati et al., 2006; Kawaguchi, 1993; Mallet et al., 2005; Sharott et al., 2009; Wilson et al., 1990). In urethane-anesthetized rats, the striatal activity is characterized by TAN with a sustained firing rate and by neurons with sporadic activity. 
A

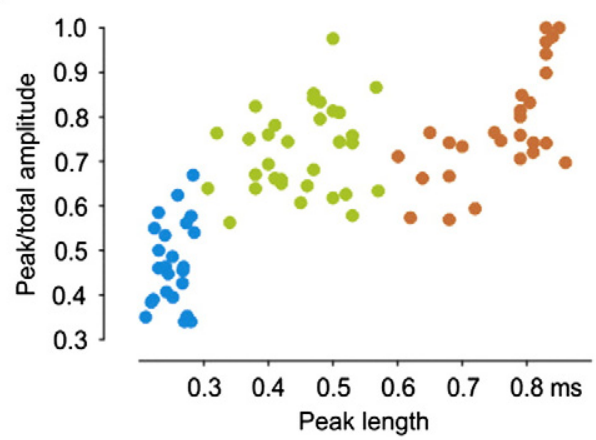

B

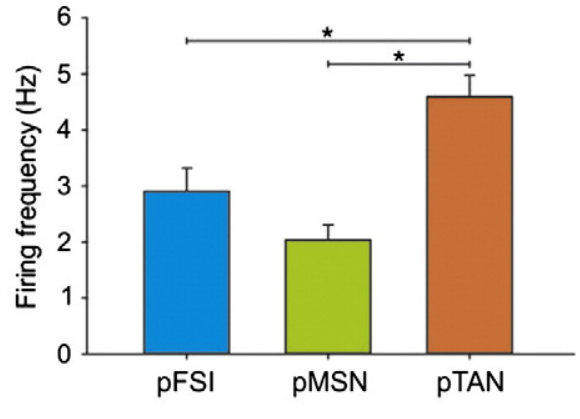

C

Spike waveform

$\mathrm{pFSI}$

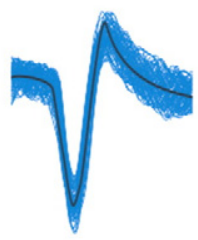

pMSN
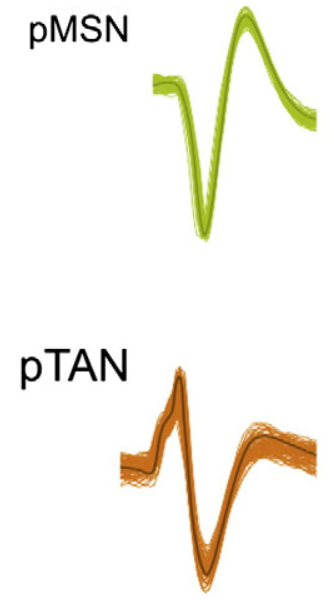

ISIH
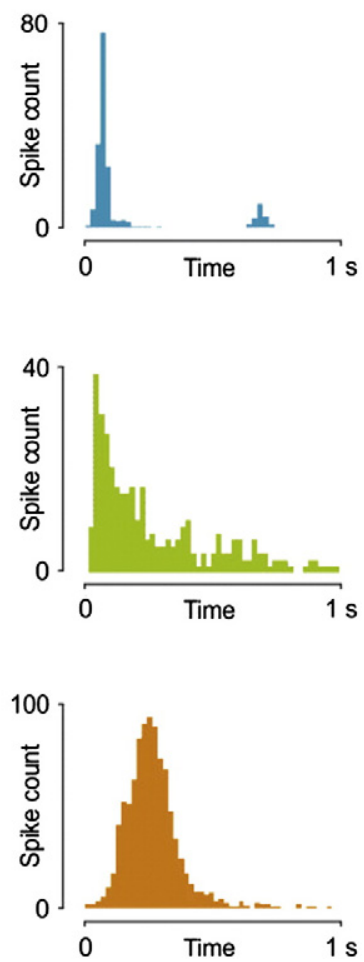

AutoCrl

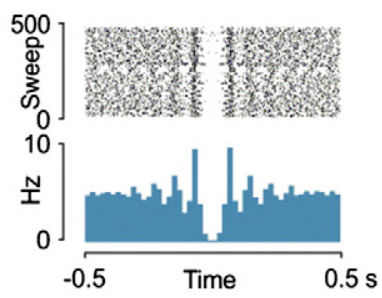

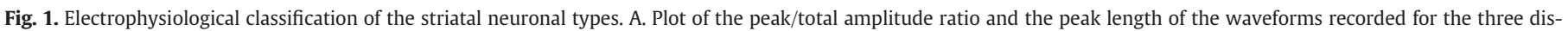

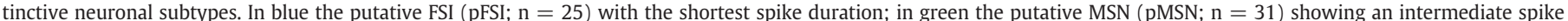

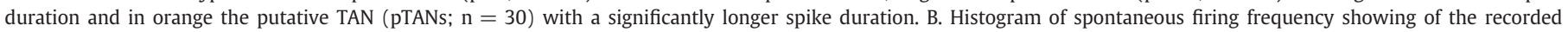

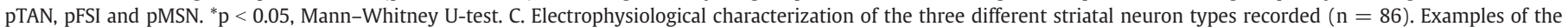
three waveforms (50 superimposed spikes) and the respective inter-spike interval histograms and autocorrelograms (500 spikes) are shown.

The waveform analysis, by plotting the peak/total amplitude ratio and the peak length of the action potentials, allowed the discrimination of three clearly distinctive neuronal subtypes (Table 1, Fig. 1): the putative TAN (pTAN), the putative MSN (pMSN) and the putative FSI (pFSI), with the latter corresponding to the sporadically active neurons. The cholinergic pTAN $(\mathrm{n}=30)$ featured a significantly longer spike duration $(0.77 \pm 0.01$ vs pMSN and pFSI, $\mathrm{p}<0.0001$, Mann-Whitney rank sum test $)$; the GABAergic pFSI $(\mathrm{n}=25)$ showed the shortest spike duration $(0.25 \pm 0.01$ vs pMSN and pTAN, $\mathrm{p}<0.0001$, Mann-Whitney rank sum test) and the GABAergic pMSN ( $\mathrm{n}=31)$ were characterized by an intermediate duration $(0.45 \pm 0.01$ vs pFSI and pTAN, $\mathrm{p}<0.0001$, Mann-Whitney rank sum test). The ratio between the peak and total amplitude was significantly lower in the pFSI $(0.46 \pm 0.37$ vs pMSN and pTAN, $\mathrm{p}<0.0001$, Mann-Whitney rank sum test) in comparison with the other two waveforms that featured similar values (pMSN
Table 1

Waveform parameters of the recorded striatal neuron subtypes.

\begin{tabular}{lll}
\hline & Peak length $(\mathrm{ms})$ & Peak/total amplitude \\
\hline pFSI $(\mathrm{n}=25)$ & $0.25 \pm 0.01$ & $0.46 \pm 0.37$ \\
& ${ }^{* *}$ vs pMSN & ${ }^{* *}$ vs pMSN \\
& ${ }^{* *}$ vs pTAN & ${ }^{* *}$ vs pTAN \\
$\operatorname{pMSN}(\mathrm{n}=31)$ & $0.45 \pm 0.01$ & $0.74 \pm 0.01$ \\
& ${ }^{* *}$ vs pFSI & ${ }^{* *}$ vs pFSI \\
& ${ }^{* *}$ vs pTAN & n.s. vs pTAN \\
pTAN $(\mathrm{n}=30)$ & $0.77 \pm 0.01$ & $0.78 \pm 0.02$ \\
& ${ }^{* *}$ vs pFSI & $* *$ vs pFSI \\
& ${ }^{* *}$ vs pMSN & n.s. vs pMSN
\end{tabular}

Data are mean \pm SEM.

${ }^{*} p<0.05,{ }^{* *} p<0.0001$, Mann-Whitney rank sum test.

n.s. $=$ non significant. 
$0.74 \pm 0.01$ and pTAN $0.78 \pm 0.02$, not significant; see Table 1, Fig. 1$)$. The mean ISI (inverse of the firing rate) of all pTAN $(0.21 \pm 0.13$, vs pMSN and pFSI, $\mathrm{p}<0.05$, Mann-Whitney rank sum test) was significantly lower than that of the pMSN $(0.47 \pm 0.08)$ and pFSI $(0.35 \pm 0.03)$, while no difference was observed between pFSI and pMSN. The firing pattern was examined by constructing the ISI histograms (ISIHs) and AutoCrls. As shown in Fig. 1, pFSI were characterized by a bimodal distribution revealing, to some extent, their tendency to burst firing corroborated by their multi-peaked AutoCrl. pMSN, instead, were characterized by an asymmetrical ISIH skewed toward shorter intervals demonstrating an irregular and sporadic firing pattern. The corresponding AutoCrl clearly showed a slow frequency oscillatory behavior that was the subject of further analysis (see below). On the contrary, pTAN predominantly manifest a unimodal ISI distribution reflecting the tonic, even if irregular, firing pattern of these cells (Fig. 1).

Changes in the activity and phase locking of striatal neurons to cortical SWA before and after TTX injection of the MFB

We examined the phase relationship between the spiking activity of single neuronal activity and the oscillatory ECoG dynamics (Fig. 2). A total of 86 neurons were recorded in the striatum before and after TTX injection into the MFB and considered eligible for subsequent analysis. The electrophysiological effect of TTX on ISI parameters are summarized in the Table 2. We conducted a population study collecting at least two neurons before and after the TTX injection from the same animal.

Before $(n=13)$ and after TTX pFSI $(n=12)$ showed a sporadic activity with a tendency to clustered firing in phase with the positive peak of SWA (Table 2). pFSI activity was affected by injection of the neurotoxin as shown by a significant increase of the mean ISI that inversely represents the firing rate $(p<0.05$ Pre-TTX vs Post-TTX, Mann-Whitney rank sum test). No major changes of the other ISI parameter were observed, that featured a quite stable pattern of activity. With respect to the ECoG, the activity of pFSI was clearly correlated to the positive phase of cortical activity. As depicted on the phase polar plots, a peak of firing probability was statistically demonstrated before TTX infusion (Friedman ANOVA, $\chi^{2} 430.52$; df 59; $p<0.0008$ ) ranging between $12^{\circ}$ and $60^{\circ}$ (Wilcoxin comparison test after Bonferroni-Holmes correction; $p<0.0008)$. TTX administration caused no considerable effect on the relationship between ECoG and pFSI (Friedman ANOVA, $\chi^{2} 416.77$, df 59, $p<0.0008$ ), although the extent of the firing probability was slightly more widely
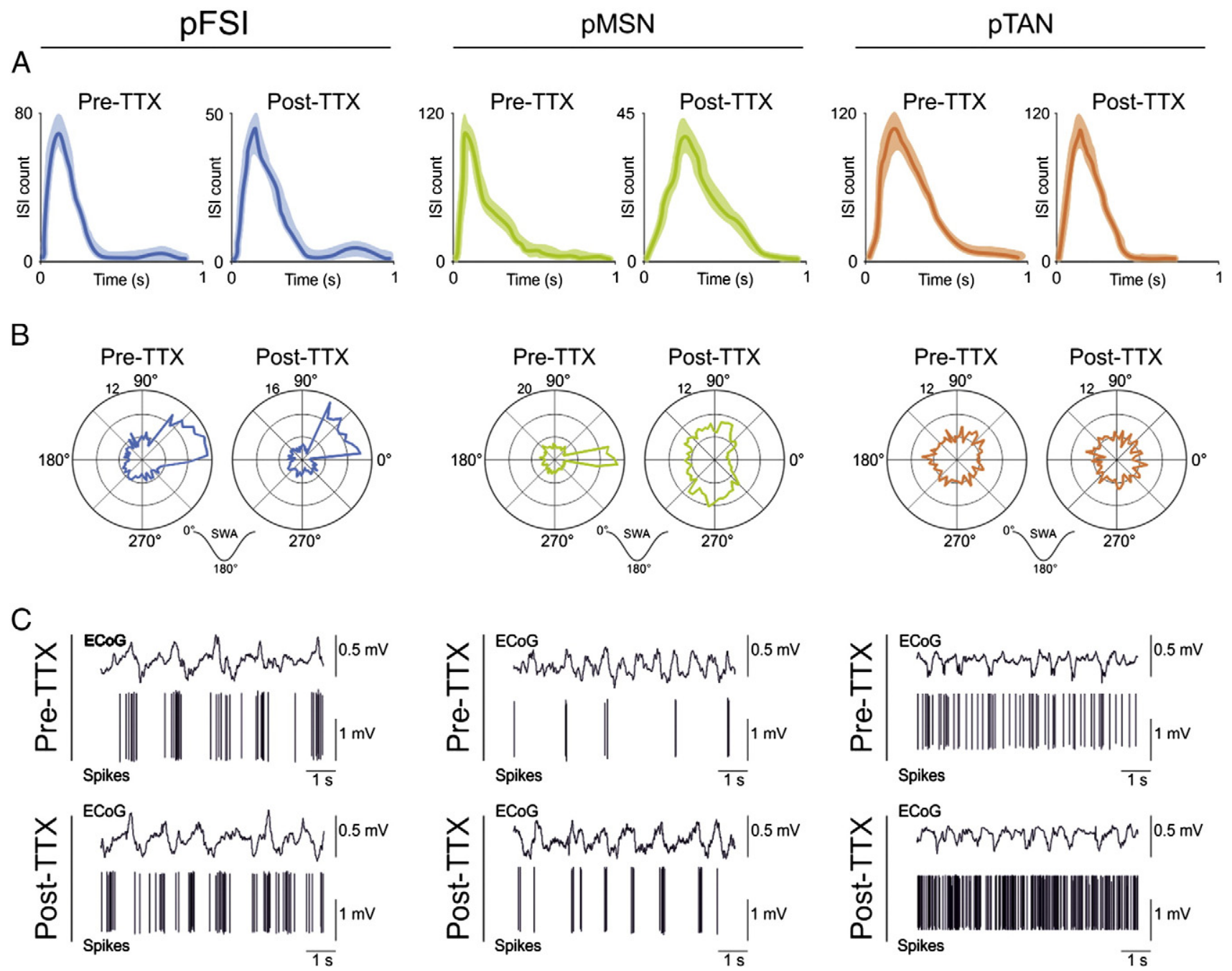

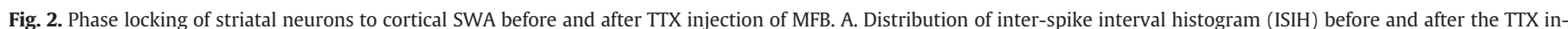

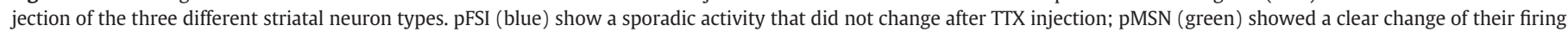

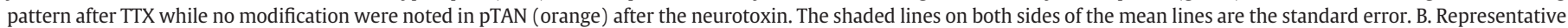

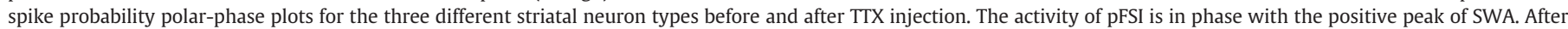

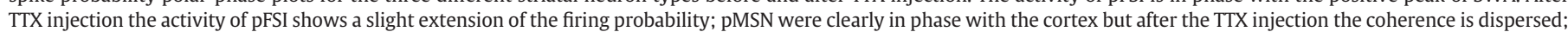

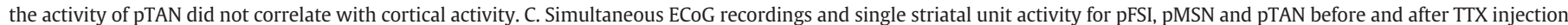
are shown. 
Table 2

Effect of TTX on ISI parameters of the recorded striatal neuron subtypes.

\begin{tabular}{|c|c|c|c|c|c|c|c|c|c|}
\hline & \multicolumn{4}{|l|}{ Pre-TTX } & & \multicolumn{4}{|l|}{ Post-TTX } \\
\hline & Mean ISI & $\mathrm{CV}$ & Skewness & Kurtosis & & Mean ISI & $\mathrm{CV}$ & Skewness & Kurtosis \\
\hline pFSI $(\mathrm{n}=13)$ & $0.35 \pm 0.03$ & $0.91 \pm 0.08$ & $2.56 \pm 0.43$ & $8.32 \pm 1.94$ & $\mathrm{pFSI}(\mathrm{n}=12)$ & $0.47 \pm 0.05^{*}$ & $0.92 \pm 0.06$ & $2.10 \pm 0.34$ & $6.42 \pm 1.79$ \\
\hline $\operatorname{pMSN}(\mathrm{n}=16)$ & $0.47 \pm 0.08$ & $1.32 \pm 0.12$ & $4.28 \pm 0.59$ & $22.6 \pm 4.40$ & $\operatorname{pMSN}(\mathrm{n}=15)$ & $0.22 \pm 0.03^{*}$ & $1.97 \pm 0.16^{*}$ & $2.82 \pm 0.28^{*}$ & $7.85 \pm 1.39^{*}$ \\
\hline pTAN $(n=12)$ & $0.21 \pm 0.13 \neq$ & $1.43 \pm 0.23$ & $3.20 \pm 0.65$ & $19.3 \pm 6.39$ & pTAN $(n=18)$ & $0.17 \pm 0.04^{*}$ & $1.50 \pm 0.10$ & $3.09 \pm 0.30$ & $14.5 \pm 3.19$ \\
\hline
\end{tabular}

Data are mean \pm SEM.

${ }^{*} p<0.05,{ }^{* *} p<0.0001$ Pre-TTX vs Post-TTX, Mann-Whitney rank sum test.

$\neq p<0.05$ pTAN vs pMSN and pFSI.

ranged (Wilcoxin comparison test after Bonferroni-Holmes correction between $6^{\circ}$ and $\left.72^{\circ} p<0.0008\right)$.

After TTX injection ( $\mathrm{n}=15$ ), we observed a clear modification of the firing pattern of the pMSN with a significant change in the distribution of ISI and a remarkable reduction of the mean ISI in parallel with significant changes of the other pattern-linked parameters $(p<0.05$, Pre-TTX vs Post-TTX, Mann-Whitney rank sum test). As depicted in Fig. 2, acute nigro-striatal DA-blockade led to a redistribution of the ISI of these neurons with a less skewed-peaked ISIH suggesting a reduced amount of grouped spikes. As far as the pMSN activity is concerned, we had striking changes before and after the TTX injection of the MFB. Specifically, the pre-TTX $(n=16)$ period was characterized by a clear-cut peak in the phase histogram ranging between $348^{\circ}$ and $12^{\circ}$ that corresponds to the positive phase of SWA (Friedman ANOVA, $\chi^{2}$ 237.08; df 59; $p<0.0008$; Wilcoxon comparison test after Bonferroni-Holmes correction). TTX-related changes of the pMSN were characterized by a dispersion of the cortico-striatal signal (Friedman ANOVA, $\chi^{2}$ 164.22; df 59; $p<0.0008)$ with no identifiable peak of firing probability $(p>0.0008$; Wilcoxon comparison test after Bonferroni-Holmes correction).

The activity of pTAN was influenced to a certain degree by TTX treatment. Although the ISI were not altered by the inactivation of MFB transmission with respect to the firing pattern, the mean ISI was significantly reduced by the TTX injection ( $p<0.05$, Pre-TTX vs Post-TTX, Mann-Whitney rank sum test). pTAN were not affected by the SWA (Friedman ANOVA, $\chi^{2} 113.75$; df 59; $p<0.0008 ; p>0.0008$; Wilcoxon comparison test after Bonferroni-Holmes correction), and their activity before and after TTX injection remained stable $(p>0.0008$; Wilcoxon comparison test after Bonferroni-Holmes correction).

\section{Discussion}

In this study we extend the analysis of the effect of the acute blockade of the MFB by TTX on the striatum that along with the STN, represents the main input structure of BG (Crutcher and DeLong, 1984). We have previously shown that TTX-induced MFB blockade dramatically changes the STN-GP interaction by increasing the cortical influence on the STN and by dictating a cortical rhythm to the GP (Galati et al., 2009). Similarly, the TTX injection into the MFB was able to change the SNr firing pattern suggesting a dysfunctional control conveyed within the cortical-BG-thalamic loop possibly causing akinesia (Galati et al., 2010). Although, the involvement of the cortical-STN hyperdirect pathway could explain the abnormal propagation of pathological rhythms in the BG (Beurrier et al., 1999; Galati et al., 2009; Nambu and Llinaś, 1994), several lines of evidence also suggested a critical role exerted by the striatum (Blume et al., 2009; Carrillo-Reid et al., 2008; Costa et al., 2006; Liang et al., 2008; Taverna et al., 2008; Tecuapetla et al., 2009; Tseng et al., 2001; Zold et al., 2012).

Striatal neurons are characterized by an extensive range of activity and waveform morphology and their spontaneous activity is markedly influenced by DA control. In agreement with previous reports (Galati et al., 2006; Mallet et al., 2006; Sharott et al., 2009, 2012) we classified the three predominant subtypes of striatal neurons on the basis of their spike waveform and firing-rate pattern. The GABAergic inhibitory MSN and the GABAergic inhibitory large aspiny FSI exhibit a low firing rate, while the GABAergic inhibitory TAN present a sustained firing under urethane anesthesia (Carrillo-Reid et al., 2009; Crutcher and DeLong, 1984; Kimura, 1992; Liang et al., 2008). Regarding the anesthesia it is important to consider the limitations of the extrapolation of the striatal firing rate; however, the rate frequencies observed were consistent with those previously described in urethane-anesthetized animals (Mallet et al., 2005, 2006; Tseng et al., 2001). Despite the possible limitations, the urethane-induced SWA allowed us to analyze how this activity spreads within striatum.

In our conditions, FSI were also characterized by a shorter spike duration and by a reduced ratio between the amplitude of the first deflection and the total amplitude. The other two neuronal populations were identified on the basis of their spontaneous activity characterized by slower and irregular activity of the MSN and a faster and more regular one of TAN (Carrillo-Reid et al., 2009; Crutcher and DeLong, 1984; Galati et al., 2006; Kimura, 1992; Liang et al., 2008; Mallet et al., 2006; Sharott et al., 2009).

Dopamine exerts a profound effect upon these neuronal populations; for instance chronic DA depletion tends to increase the spontaneous activity of the MSN (Day et al., 2006; Liang et al., 2008; Uhlhaas and Singer, 2006; Zold et al., 2012), that was linked to an augmented glutamatergic tone (Galarraga et al., 1986; Goldberg et al., 2004; Raz et al., 1996; Tseng et al., 2001) and to reducing the feed-forward FSI-mediated inhibition (Bracci et al., 2002; Magill et al., 2006). In our study we observed a pronounced reconfiguration of the striatal microcircuit following the blockade of the nigro-striatal interplay. The acute DA-denervated model allowed us to examine what occurs immediately after the functional interference of the SNc DA signaling flow to the striatum. Regardless of cortical activity, TTX injection profoundly modified the activity of striatal neuronal subtypes. In accordance with the literature on chronically denervated animals, we found a mean overall increase of the firing rate of putative striatal projection neurons(Flores-Barrera et al., 2010; Hernandez-Lopez et al., 2000; Mallet et al., 2006; Shen et al., 2007; Surmeier et al., 2010; West and Grace, 2002). Striatal cholinergic interneurons undergo an increase in excitability in chronically dopaminedepleted animals (Salin et al., 2009). Accordingly, a study in rat brain slices of acutely dopamine-depleted animals demonstrated that MSN and cholinergic interneurons become more excitable, while GABAergic interneurons do not significantly change their firing rate (Fino et al., 2007).

So far, under physiological conditions the activity of striatal neurons appears strongly influenced by the cortical activation state (Mallet et al., 2005; Sharott et al., 2012). MSN change their firing rates and patterns mainly according to SWA and interneuronal activity. As recently demonstrated by Sharott et al. (2012), histologically identified cholinergic and GABAergic parvalbumine positive $(\mathrm{PV}+)$ interneurons show higher firing rates during the cortical activation state rather than during SWA, whereas the average firing of the MSN population seems to be unaffected by cortical activity. The physiological activity of the striatal projection neurons as well as the FSI is already in phase with the SWA (Mallet et al., 2005; Tseng et al., 2001; present observations). The firing probability of the MSN achieved its maximum during the peak of SWA firing that coincides with the up-state membrane potential of the MSN (Tseng et al., 2001). Similarly to the physiological condition, the chronic denervated 
state did not alter this cortico-striatal coherence, although the overall activity was augmented (Tseng et al., 2001). Other authors suggested that the DA depletion in 6-OHDA-lesioned rats increases the ability of MSN to escape from the feed-forward inhibition by FSI, with the unchanged FSI activity in this experimental setting resulting in a further dyscontrol of MSN activity (Mallet et al., 2005). We found that acute DA denervation led to a tendency of the pMSN to discharge with an enlarged time window with respect to the SWA, breaking up the usual cortico-striatal inter-talk. Furthermore, the acute interference with nigro-striatal signaling by TTX unmasked change of the interplay between SWA and FSI activity with a slight but significant more widespread phase correlation. FSI are considered the GABAergic PV + striatal interneurons that target the MSN in close proximity to their soma and receive cortical input (Kawaguchi, 1993; Mallet et al., 2005; Ramanathan et al., 2002). Therefore, we suppose that slight changes of FSI activity strongly affect the MSN. The acute DA depletion protocol allowed us to reveal the mechanism of action of DA upon the FSI-MSN interaction in vivo. Indeed, in vitro studies have shown that DA directly depolarizes FSI via D1-like receptors and decreases the inhibitory synaptic input to these cells via presynaptic D2-like receptors (Ballion et al., 2009; Bracci et al., 2002; Centonze et al., 2003). In agreement, we found that the TTX-mediated effect on FSI was characterized not only by a significant decrease of the firing rate, but also by a slightly more widespread firing probability during the active component of SWA. Thus, as opposed to what was observed in the chronic animal model (Mallet et al., 2006), we found a clear-cut decrease of FSI inhibitory tone upon the MSN with a probable escape of these neurons from cortical control. The role of TAN can possibly rather be linked to the thalamo-striatal pathway. Indeed, cholinergic neurons are quite independent of cortical control (i.e. less affected by SWA), whilst showing an augmented activity in Parkinsonian state, in both the chronic (Sanchez et al., 2011) and acute condition (Fino et al., 2007; present data). We hypothesize that they maintain a surrogate role as feedback circuit not necessarily involving the cortex. In fact, the thalamic gating of cortico-striatal signalling by TAN works in a way that activation of thalamo-striatal axons induces a burst of spikes in striatal cholinergic interneurons followed by a pause lasting more than half a second (Ding et al., 2010). This patterned interneuron activity suggests that the transient presynaptic suppression of cortical input to MSN is able to control the end of a movement. Thus, this differential regulation of the cortico-striatal circuitry operated by TAN provides a neural substrate for attentional shifts and cessation of ongoing motor activity with the appearance of salient environmental stimuli. This mechanism could hypothetically also play a role in the clinical observation of PD patients, apparently akinetic or even stuck to the floor due to a desperate freezing, are suddenly capable of escaping from a fire. Therefore, in functional terms, the PD patients' inability to walk autonomously might be due to the fact that the striatum is unable to direct, discriminate and convey the "go" signal. Their surprising ability to run away could be explained by the surrogate order of the thalamo-TAN pathway coming into play, activated by the sudden attentional shift. However, an extensive degeneration of thalamic relay neurons, the major source of the thalamo-striatal system, may in fact occur in PD representing a major obstacle when transferring data from rodent models, whose degeneration is by definition limited to nigro-fugal elements.

\section{Conclusions}

Altogether, the TTX-induced blockade of the nigro-striatal interplay led to an impaired and poorly timed cortical-driven pFSI activity with a consequent impairment of the otherwise tightly controlled activity of the striatal projection neurons. Thus, pMSN tended to fire irrespectively of the cortical activity and were less affected by the pFSI. We therefore suggest that during acute DA depletion the striatal projection neurons are less phase-locked to the SWA. This dispersion of pMSN discharges immediately after the TTX infusion indicates that the principal output cells lose their dependency on cortical inputs. The striking change of pFSI discharges supports the contention that the interaction between pFSI-pMSN is the critical message disrupted. On the one hand this does not imply an exclusion of the striatum in shaping BG circuitry, but on the other hand suggests that the striatum has probably lost the ability to convey messages through selective (i.e. direct vs indirect) pathways. Thus its involvement in cortical-BG synchronization seems questionable, making the participation of the hyperdirect pathway more likely as previously discussed (Galati et al., 2009). We cannot exclude, however, that the timing dysfunction of different neurons, mainly the pFSI and pMSN, might also alter downstream network activities rendering them more susceptible to cortical rhythms.

In summary, our data improve the understanding of the effect of dopaminergic dysfunction on the striatal network and encoding of cortical information and on the propagation of rhythmic activity in Parkinsonian conditions.

\section{Acknowledgments}

We thank the Fondazione per lo studio delle malattie neurodegenerative delle persone adulte e dell'anziano del Ticino for the financial support. We are also grateful to Franco Lavaroni, Teresa Procopio, Marta Pace and Agnese Salvadè for their technical assistance.

\section{References}

Ballion, B., Frenois, F., Zold, C.L., Chetrit, J., Murer, M.G., Gonon, F., 2009. D2 receptor stimulation, but not D1, restores striatal equilibrium in a rat model of Parkinsonism. Neurobiol. Dis. 35, 376-384.

Beurrier, C., Congar, P., Bioulac, B., Hammond, C., 1999. Subthalamic nucleus neurons switch from single-spike activity to burst-firing mode. J. Neurosci. 19, 599-609.

Blume, S.R., Cass, D.K., Tseng, K.Y., 2009. Stepping test in mice: a reliable approach in determining forelimb akinesia in MPTP-induced Parkinsonism. Exp. Neurol. 219, 208-211.

Bracci, E., Centonze, D., Bernardi, G., Calabresi, P., 2002. Dopamine excites fast-spiking interneurons in the striatum. J. Neurophysiol. 87, 2190-2194.

Brown, P., 2003. Oscillatory nature of human basal ganglia activity: relationship to the pathophysiology of Parkinson's disease. Mov. Disord. 18, 357-363.

Carrillo-Reid, L., Tecuapetla, F., Tapia, D., Hernández-Cruz, A., Galarraga, E., DruckerColin, R., Bargas, J., 2008. Encoding network states by striatal cell assemblies. J. Neurophysiol. 99, 1435-1450.

Carrillo-Reid, L., Tecuapetla, F., Vautrelle, N., Hernández, A., Vergara, R., Galarraga, E. Bargas, J., 2009. Muscarinic enhancement of persistent sodium current synchronizes striatal medium spiny neurons. J. Neurophysiol. 102, 682-690.

Centonze, D., Grande, C., Usiello, A., Gubellini, P., Erbs, E., Martin, A.B., Pisani, A., Tognazzi, N., Bernardi, G., Moratalla, R., Borrelli, E., Calabresi, P., 2003. Receptor subtypes involved in the presynaptic and postsynaptic actions of dopamine on striatal interneurons. J. Neurosci. 23, 6245-6254.

Costa, R.M., Lin, S.C., Sotnikova, T.D., Cyr, M., Gainetdinov, R.R., Caron, M.G., Nicolelis, M.A., 2006. Rapid alterations in corticostriatal ensemble coordination during acute dopamine-dependent motor dysfunction. Neuron 52, 359-369.

Crutcher, M.D., DeLong, M.R., 1984. Single cell studies of the primate putamen. I. Functional organization. Exp. Brain Res. 53, 233-243.

Day, M., Wang, Z., Ding, J., An, X., Ingham, C.A., Shering, A.F., Wokosin, D., Ilijic, E., Sun, Z., Sampson, A.R., Mugnaini, E., Deutch, A.Y., Sesack, S.R., Arbuthnott, G.W., Surmeier, D.J., 2006. Selective elimination of glutamatergic synapses on striatopallidal neurons in Parkinson disease models. Nat. Neurosci. 9, 251-259.

Ding, J.B., Guzman, J.N., Peterson, J.D., Goldberg, J.A., Surmeier, D.J., 2010. Thalamic gating of corticostriatal signaling by cholinergic interneurons. Neuron 67, 294-307.

Eusebio, A., Thevathasan, W., Doyle Gaynor, L., Pogosyan, A., Bye, E., Foltynie, T., Zrinzo, L., Ashkan, K., Aziz, T., Brown, P., 2011. Deep brain stimulation can suppress pathological synchronisation in parkinsonian patients. J. Neurol. Neurosurg. Psychiatry $82,569-573$.

Fino, E., Glowinski, J., Venance, L., 2007. Effects of acute dopamine depletion on the electrophysiological properties of striatal neurons. Neurosci. Res. 58, 305-316.

Flores-Barrera, E., Vizcarra-Chacón, B.J., Tapia, D., Bargas, J., Galarraga, E., 2010. Different corticostriatal integration in spiny projection neurons from direct and indirect pathways. Front. Syst. Neurosci. 10 (4), 15

Fuentes, R., Petersson, P., Siesser, W.B., Caron, M.G., Nicolelis, M.A., 2009. Spinal cord stimulation restores locomotion in animal models of Parkinson's disease. Science 323, 1578-1582.

Galarraga, E., Corsi-Cabrera, M., Sangri, M., 1986. Reduction in paradoxical sleep after Ldopa administration in rats. Behav. Neural Biol. 46, 249-256.

Galati, S., Mazzone, P., Fedele, E., Pisani, A., Peppe, A., Pierantozzi, M., Brusa, L., Tropepi, D., Moschella, V., Raiteri, M., Stanzione, P., Bernardi, G., Stefani, A., 2006. Biochemical and electrophysiological changes of substantia nigra pars reticulata driven by subthalamic stimulation in patients with Parkinson's disease. Eur. J. Neurosci. 23 2923-2928. 
Galati, S., D'Angelo, V Scarnati, E Stanzione, P., Martorana, A., Procopio, T., Sancesario, G., Stefani, A., 2008. In vivo electrophysiology of dopamine-denervated striatum: focus on the nitric oxide/cGMP signaling pathway. Synapse 62, 409-420.

Galati, S., Stanzione, P., D'Angelo, V., Fedele, E., Marzetti, F., Sancesario, G., Procopio, T., Stefani, A., 2009. The pharmacological blockade of medial forebrain bundle induces an acute pathological synchronization of the cortico-subthalamic nucleus-globus pallidus pathway. J. Physiol. 587, 4405-4423.

Galati, S., D'Angelo, V., Olivola, E., Marzetti, F., Di Giovanni, G., Stanzione, P., Stefani, A. 2010. Acute inactivation of the medial forebrain bundle imposes oscillations in the SNr: a challenge for the 6-OHDA model? Exp. Neurol. 225, 294-301.

Goldberg, J.A., Rokni, U., Boraud, T., Vaadia, E., Bergman, H., 2004. Spike synchronization in the cortex/basal-ganglia networks of Parkinsonian primates reflects global dynamics of the local field potentials. J. Neurosci. 24, 6003-6010.

Hammond, C., Bergman, H., Brown, P., 2007. Pathological synchronization in Parkinson's disease: networks, models and treatments. Trends Neurosci. 30, 357-364.

Hernandez-Lopez, S., Tkatch, T., Perez-Garci, E., Galarraga, E., Bargas, J., Hamm, H, Surmeier, D.J., 2000. D2 dopamine receptors in striatal medium spiny neurons reduce L-type Ca2 + currents and excitability via a novel PLC[beta]1-IP3-calcineurinsignaling cascade. J. Neurosci. 20 (24), 8987-8995 (Dec 15).

Jáidar, O., Carrillo-Reid, L., Hernández, A., Drucker-Colín, R., Bargas, J., Hernández-Cruz, A., 2010. Dynamics of the Parkinsonian striatal microcircuit: entrainment into a dominant network state. J. Neurosci. 30, 11326-11336.

Kawaguchi, Y., 1993. Physiological, morphological, and histochemical characterization of three classes of interneurons in rat neostriatum. J. Neurosci. 13, 4908-4923.

Kimura, M., 1992. Behavioral modulation of sensory responses of primate putamen neurons. Brain Res. 578, 204-214

Kühn, A.A., Kupsch, A., Schneider, G.H., Brown, P., 2006. Reduction in subthalamic 8-35 Hz oscillatory activity correlates with clinical improvement in Parkinson's disease. Eur. J. Neurosci. 23, 1956-1960.

Liang, L., DeLong, M.R., Papa, S.M., 2008. Inversion of dopamine responses in striata medium spiny neurons and involuntary movements. J. Neurosci. 28, 7537-7547.

Magill, P.J., Bolam, J.P., Bevan, M.D., 2000. Relationship of activity in the subthalamic nucleus-globus pallidus network to cortical electroencephalogram. J. Neurosci. 20, 820-833.

Magill, P.J., Bolam, J.P., Bevan, M.D., 2001. Dopamine regulates the impact of the cerebral cortex on the subthalamic nucleus-globus pallidus network. Neuroscience 106, 313-330.

Magill, P.J., Pogosyan, A., Sharott, A., Csicsvari, J., Bolam, J.P., Brown, P., 2006. Changes in functional connectivity within the rat striatopallidal axis during global brain activation in vivo. J. Neurosci. 26, 6318-6329.

Mallet, N., Le Moine, C., Charpier, S., Gonon, F., 2005. Feed-forward inhibition of projection neurons by fast-spiking GABA interneurons in the rat striatum in vivo. $\mathrm{J}$ Neurosci. 25, 3857-3869.

Mallet, N., Ballion, B., Le Moine, C., Gonon, F., 2006. Cortical inputs and GABA interneurons imbalance projection neurons in the striatum of parkinsonian rats. J. Neurosci. 26, 3875-3884

McCarthy, M.M., Moore-Kochlacs, C., Gu, X., Boyden, E.S., Han, X., Kopell, N., 2011. Striatal origin of the pathologic beta oscillations in Parkinson's disease. Proc. Natl. Acad. Sci. U. S. A. 108, 11620-11625.

Nambu, A., Llinaś, R., 1994. Electrophysiology of globus pallidus neurons in vitro. J. Neurophysiol. 72, 1127-1139.

Plenz, D., Kitai, S.T., 1999. A basal ganglia pacemaker formed by the subthalamic nucleus and external globus pallidus. Nature 400, 677-682.
Ramanathan, S., Hanley, J.J., Deniau, J.M., Bolam, J.P., 2002. Synaptic convergence of motor and somatosensory cortical afferents onto GABAergic interneurons in the rat striatum. J. Neurosci. 22, 8158-8169.

Raz, A., Feingold, A., Zelanskaya, V., Vaadia, E., Bergman, H., 1996. Neuronal synchronization of tonically active neurons in the striatum of normal and parkinsonian primates. J. Neurophysiol. 76, 2083-2088.

Salin, P., López, I.P., Kachidian, P., Barroso-Chinea, P., Rico, A.J., Gómez-Bautista, V., Coulon, P., Kerkerian-Le Goff, L., Lanciego, J.L., 2009. Changes to interneuron-driven striatal microcircuits in a rat model of Parkinson's disease. Neurobiol. Dis. 34, 545-552.

Sanchez, G., Rodriguez, M.J., Pomata, P., Rela, L., Murer, M.G., 2011. Reduction of an afterhyperpolarization current increases excitability in striatal cholinergic interneurons in rat parkinsonism. J. Neurosci. 31, 6553-6564.

Sharott, A., Magill, P.J., Bolam, J.P., Brown, P., 2005. Directional analysis of coherent oscillatory field potentials in the cerebral cortex and basal ganglia of the rat. J. Physiol. 562, 951-963.

Sharott, A., Moll, C.K., Engler, G., Denker, M., Grün, S., Engel, A.K., 2009. Different subtypes of striatal neurons are selectively modulated by cortical oscillations. J. Neurosci. 29, 4571-4585.

Sharott, A., Doig, N.M., Mallet, N., Magill, P.J., 2012. Relationships between the firing of identified striatal interneurons and spontaneous and driven cortical activities in vivo. J. Neurosci. 32, 13221-13236.

Shen, W., Tian, X., Day, M., Ulrich, S., Tkatch, T., Nathanson, N.M., Surmeier, D.J., 2007. Cholinergic modulation of Kir2 channels selectively elevates dendritic excitability in striatopallidal neurons. Nat. Neurosci. 10, 1458-1466.

Steriade, M., Nuñez, A., Amzica, F., 1993. A novel slow $(<1 \mathrm{~Hz})$ oscillation of neocortical neurones in vivo: depolarizing and hyperpolarizing components. J. Neurosci. 13, 3252-3265.

Surmeier, D.J., Shen, W., Day, M., Gertler, T., Chan, S., Tian, X., Plotkin, J.L., 2010. The role of dopamine in modulating the structure and function of striatal circuits. Prog. Brain Res. 183, 149-167.

Tang, K., Low, M.J., Grandy, D.K., Lovinger, D.M., 2001. Dopamine-dependent synaptic plasticity in striatum during in vivo development. Proc. Natl. Acad. Sci. U. S. A. 98, 1255-1260.

Taverna, S., Ilijic, E., Surmeier, D.J., 2008. Recurrent collateral connections of striatal medium spiny neurons are disrupted in models of Parkinson's disease. J. Neurosci. 28, 5504-5512.

Tecuapetla, F., Koós, T., Tepper, J.M., Kabbani, N., Yeckel, M.F., 2009. Differential dopaminergic modulation of neostriatal synaptic connections of striatopallidal axon collaterals. J. Neurosci. 29, 8977-8990.

Tseng K.Y., Kasanetz, F., Kargieman, L., Riquelme, L. A, Murer, M.G, 2001. Cortical slow oscillatory activity is reflected in the membrane potential and spike trains of striatal neurons in rats with chronic nigrostriatal lesions. J. Neurosci. 21, 6430-6439.

Uhlhaas, P.J., Singer, W., 2006. Neural synchrony in brain disorders: relevance for cognitive dysfunctions and pathophysiology. Neuron 52, 155-168.

West, A.R., Grace, A.A., 2002. Opposite influences of endogenous dopamine D1 and D2 receptor activation on activity states and electrophysiological properties of striatal neurons: studies combining in vivo intracellular recordings and reverse microdialysis. J. Neurosci. 22, 294-304.

Wilson, C.J., Chang, H.T., Kitai, S.T., 1990. Firing patterns and synaptic potentials of identified giant aspiny interneurons in the rat neostriatum. J. Neurosci. 10, 508-519.

Zold, C.L., Escande, M.V., Pomata, P.E., Riquelme, L.A., Murer, M.G., 2012. Striatal NMDA receptors gate cortico-pallidal synchronization in a rat model of Parkinson's disease. Neurobiol. Dis. 47, 38-48. 